\title{
Hepatitis C Virus Core Protein Is Efficiently Released into the Culture Medium in Insect Cells
}

\author{
Soo Ho Choi, So Yeon Kim, Kyu Jin Park, Yeon Joo Kim and Soon Bong Hwang* \\ Ilsong Institute of Life Science, Hallym University, Chuncheon 200-702, Korea
}

Received 10 June 2004, Accepted 24 July 2004

\begin{abstract}
Hepatitis $\mathrm{C}$ virus (HCV) is a causal agent of the chronic liver infection. To understand HCV morphogenesis, we studied the assembly of $\mathrm{HCV}$ structural proteins in insect cells. We constructed recombinant baculovirus expression vectors consisting of either $\mathrm{HCV}$ core alone, core-E1, or core-E1-E2. These structural proteins were expressed in insect cells and were examined to assemble into particles. Neither core-E1 nor core-E1-E2 was capable of assembling into virus-like particles (VLPs). It was surprising that the core protein alone was assembled into core-like particles. These particles were released into the culture medium as early as 2 days after infection. In our system, HCV structural proteins including envelope proteins did not assemble into VLPs. Instead, the core protein itself has the intrinsic capacity to assemble into amorphous core-like particles. Furthermore, released core particles were associated with HCV RNA, indicating that core proteins were assembled into nucleocapsids. These results suggest that HCV may utilize a unique core release mechanism to evade the hosts defense mechanism, thus contributing to the persistence of $\mathrm{HCV}$ infection.
\end{abstract}

Keywords: Core-like particles, Hepatitis C virus, Nucleocapsid, Virus assembly, Virus-like particles

\section{Introduction}

Hepatitis $\mathrm{C}$ virus (HCV), a member of the Flaviviridae

\footnotetext{
Abbreviations: HCV, hepatitis C virus; VLP, virus-like particle; Sf9, Spodoptera frugiperda; ER, endoplasmic reticulum; m.o.i., multiplicity of infection
}

*To whom correspondence should be addressed.

Tel: 82-31-380-1732; Fax: 82-31-384-5395

E-mail: sbhwang@hallym.ac.kr family, is an important human pathogen associated with chronic hepatitis and hepatocellular carcinoma (Saito et al., 1990; Shimotohno, 1993). HCV contains a positive-sense, single-stranded RNA genome of approximately $9.6 \mathrm{~kb}$ (Choo et al., 1989; Kato et al., 1990; Miller and Purcell, 1990; Inchauspe et al., 1991; Takamizawa et al., 1991). The genome contains a single open reading frame that is flanked by untranslated regions (UTR) at both the $5^{\prime}$ and 3 ' termini. The highly conserved 5' UTR contains an internal ribosome entry site (IRES) that mediates the CAP-independent translation of the viral RNA (Wang and Siddiqui, 1995). The viral genome encodes a single polyprotein precursor of 3,010 to 3,030 amino acids, which is proteolytically processed by both hostand virus-encoded proteases into 10 distinct structural and nonstructural proteins (Hijikata et al., 1991; Grakoui et al., 1993; Lin et al., 1994). The $\mathrm{NH}_{2}$-terminal portion of the polyprotein encodes structural proteins including core, and two envelope proteins, E1 and E2/p7 and the carboxyl terminus comprises nonstructural proteins. Although both biochemical and functional properties of many structural and nonstructural proteins have been well characterized, studies on virion morphogenesis and viral replication have been hampered by the lack of an efficient tissue culture system.

$\mathrm{HCV}$ is an enveloped virus and virion size is between 30 to $60 \mathrm{~nm}$. Virus-like particles have been identified in thin-section electron microscopy of in vitro cultured cells. However, the mechanism of $\mathrm{HCV}$ virion assembly is not understood because the expression of the HCV structural gene in mammalian cells generates no detectable virion particles. In the present study, we demonstrate that core protein alone undergoes self-assembly into particle-like structure in recombinant baculovirus-infected insect cells. Neither E1 nor E2 envelope protein was required for particle assembly. In our system, core proteins alone were sufficient for assembly into core-like particles. The availability of large amounts of corelike particles will provide us a system to study viral morphogenesis and to develop a serological test for the diagnosis of $\mathrm{HCV}$ infection. 


\section{Materials and Methods}

Construction of expression vectors $\mathrm{HCV}$ cDNA sequence (genotype $1 b$ ) corresponding to the structural proteins (core, E1, E2) was subcloned into the baculovirus shuttle vector, pVL941 as described previously (Hwang et al., 1997). Briefly, cDNAs corresponding to the both wild type and mutant forms of $\mathrm{HCV}$ structural genes were amplified by polymerase chain reaction (PCR) using Taq DNA polymerase (Roche, Mannheim, Germany). Each primer contains a BamHI or a BglII site and a protein initiation codon (ATG) at the front, and a stop codon (TGA) plus a $B a m \mathrm{H} 1$ or a $B g / I I$ endonuclease site at the end. The amplified PCR product was gel-purified and digested with either BamH1 or BglII and inserted into the BamHI site of the pVL941 vector behind polyhedrin promoter.

Production of recombinant baculoviruses Spodoptera frugiperda (Sf9) insect cells were cotransfected with wild type baculovirus (Autographa Californica nuclear polyhedrosis virus, AcNPV) DNA and each recombinant transfer vector DNA as described previously (Hwang et al., 1997). Culture supernatants were harvested at day 4 posttransfection and used for plaque assays. Each virus isolated from a plaque was used to infect Sf9 cells to amplify recombinant viruses. Protein expressions were examined either by SDS-PAGE and Coomassie blue staining or by Western blot analysis.

Purification of core-like particles Sf9 cells were infected with recombinant baculoviruses at a multiplicity of infection (m.o.i.) of 3 and incubated at $27^{\circ} \mathrm{C}$. The culture supernatant was collected at 3 days after infection and cell debris were removed by centrifugation at 3,500 rpm for $15 \mathrm{~min}$. Supernatant was further subjected to centrifugation at $12,000 \mathrm{rpm}$ for $30 \mathrm{~min}$ to eliminate the baculoviruses. The supernatant was pelleted through $30 \%$ sucrose cushion (wt/vol in PBS) for $90 \mathrm{~min}$ at 27,000 rpm using a SW 28 rotor. For equilibrium density gradient sedimentation, the pellet was resuspened in a solution of $\mathrm{CsCl}(1.32 \mathrm{~g} / \mathrm{ml})$ and centrifuged at $48,000 \mathrm{rpm}$ for $24 \mathrm{~h}$ in a SW 50.1 rotor. Ten fractions were diluted in PBS, pelleted, and subjected to Western blot analysis using HCV patient sera.

Western blot analysis Recombinant baculovirus-infected Sf9 cells were harvested at 3 days after infection and washed twice in PBS. Either cell lysates or purified particles were separated by SDS-PAGE and transferred to a nitrocellulose membrane for $1 \mathrm{~h}$. The membrane was incubated with $\mathrm{HCV}$ patient serum and proteins were visualized as previously described (Park et al., 2000; Kim et al., 2004).

HCV RNA detection by RT-PCR Culture supernatants were harvested from cells infected with recombinant viruses expressing either HCV core or HCV core-E1-E2 proteins. Culture supernatants were subjected to centrifugation using $30 \%$ sucrose cushion as described above. RNA was extracted from the pellet and reverse transcribed by AMV reverse transcriptase (Promega, Madison, USA) using the primer 5'-GGAAGATCTTCAAGCAGAAACTGG GGT-3', which corresponds the C-terminal end of the core. The cDNA was amplified with this primer and the sense primer 5'CCGCTCGAGGATGAGCACAAATCC-3' of the HCV core.

\section{Results}

Expression of HCV structural proteins in insect cells In order to understand the HCV morphogenesis, we generated recombinant baculoviruses encoding various parts or portions of HCV structural proteins (Fig. 1) and used them to infect insect cells with low m.o.i. $\left(10^{3}\right)$. Cell lysates were prepared at 3 days after infection and analyzed for protein expression. As shown in Fig. 2A, all recombinant baculovirus-infected cells expressed the corresponding $\mathrm{HCV}$ structural proteins. As reported previously, core protein consisted of two bands, 19 $\mathrm{kDa}$ and $21 \mathrm{kDa}$ (Hwang, 1998). Protein expressions of both E1 and E2 were verified by immunoblotting with monoclonal anti-E1 and E2 antibodies, respectively (data not shown). It was interesting that $\mathrm{C}$-terminus of $\mathrm{E} 1$ and $\mathrm{p} 7$ exerted the inhibitory effect on overall expression levels of structural proteins (Fig. 2A, lanes 4 and 6). Furthermore, E1 protein was barely detectable when it was expressed as a fusion to the E2 envelope protein (Fig. 2A, lane 5). It is uncertain why E1 expression is so low in this construct. In fact, many $\mathrm{HCV}$ patient sera showed the similar results (data not shown).

Core-like particles but not VLPs were produced from the structural gene Since all constructs were verified to express correct proteins, we examined if any construct would produce VLP. We first examined intracellular VLPs using electron microscopy. However, no VLPs were detected in recombinant baculovirus-infected cells. Based on other group's findings (Luo et al., 1990; Jiang et al., 1992; Crawford et al., 1994), we then examined cell culture supernatant to test whether $\mathrm{HCV}$ structural proteins were released into the medium. Sf9 cells were infected with recombinant baculoviruses and cell culture supernatant was harvested at day 3 postinfection. The supernatant was pelleted through a $30 \%$ sucrose cushion and

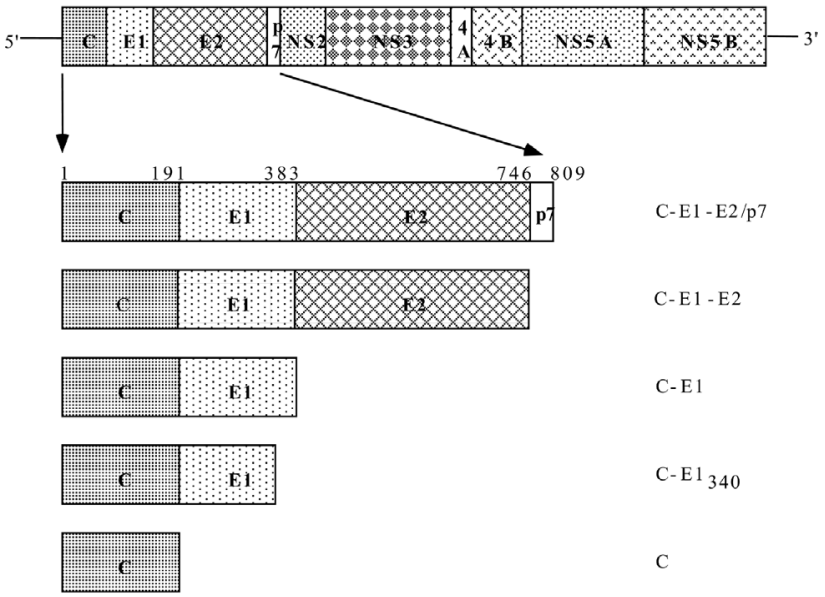

Fig. 1. Schematic diagram of HCV structural protein constructs used in baculovirus expression system. cDNA corresponding to the HCV structural gene was subcloned into the BamHI site of the transfer vector pVL941 behind polyhedrin promoter. Recombinant baculoviruses expressing HCV structural proteins were produced as described in Materials and Methods. 
A

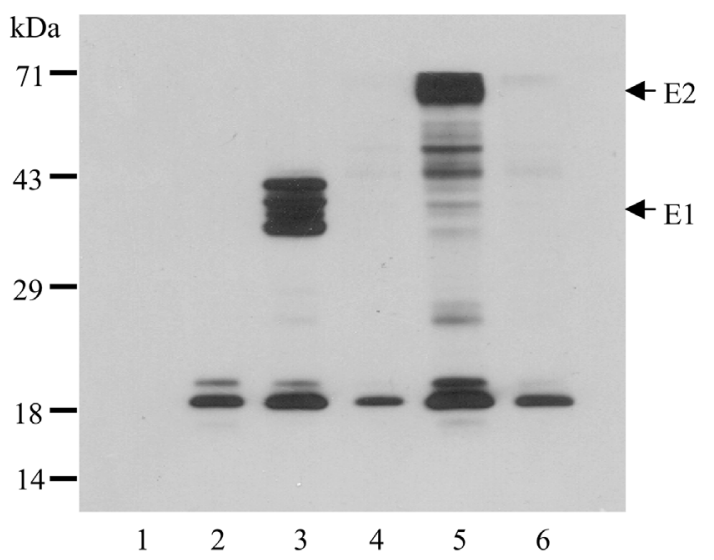

B

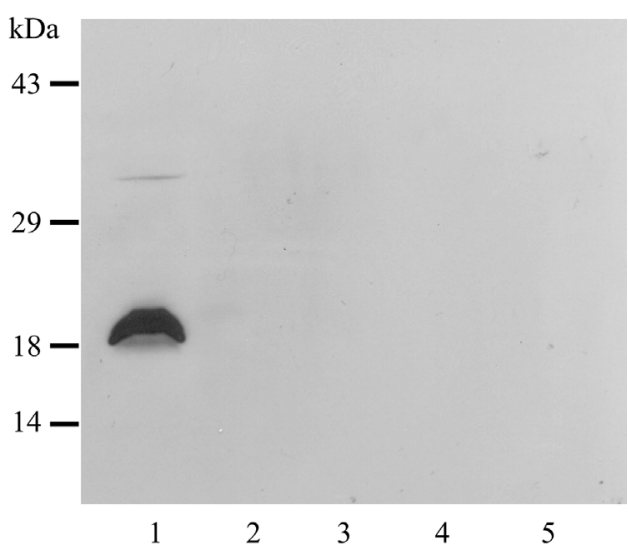

Fig. 2. Core-like particles but not VLPs were produced from the structural gene. (A) Expression of HCV structural proteins in insect cells. Sf9 cells were infected with recombinant baculoviruses expressing HCV structural proteins and were harvested at day 3 postinfection. Cell lysates were separated by SDS-containing polyacrylamide gel electrophoresis, transferred to a nitrocellulose membrane. Proteins were detected by Western blotting using HCV patient sera. Lane 1, mock-infected; lane 2, core; lane 3, C-E1 340; lane 4, C-E1; lane 5, C-E1-E2; lane 6, C-E1-E2 (p7). The results shown are representative of three independent experiments. (B) The culture medium from recombinant baculovirus-infected insect cells was centrifuged at 3,500 rpm for 15 min to remove cell debris. Supernatant was further centrifuged at $12,000 \mathrm{rpm}$ for $30 \mathrm{~min}$ to remove recombinant baculoviruses. The resultant supernatant was then pelleted through a $30 \%$ sucrose cushion for $90 \mathrm{~min}$ at $27,000 \mathrm{rpm}$. The pellet was dissolved in sample buffer and analyzed by Western blotting using HCV patient sera. Lane 1, core; lane 2, C-E1340; lane 3, C-E1; lane 4, C-E1-E2; lane 5, C-E1-E2 (p7).

examined for VLPs using an HCV patient serum. The result showed that only the supernatant collected from the coreexpressing cells contained the particles that reacted with an $\mathrm{HCV}$ patient serum (Fig. 2B, lane 1) and thus might represent core-like particles. However, neither core-E1- nor core-E1-E2expressing culture medium contained VLPs. We further looked for VLPs in the culture supernatant harvested from envelope-expressing cells at day 4 postinfection. Although cytolysis started to occur at day 4 postinfection (Hwang et al., 1998), we were unable to detect VLPs. To further examine the presence of VLPs, we used $20 \%$ sucrose cushion, instead of $30 \%$, to pellet the culture supernatant. Nevertheless, only core protein, without envelope proteins, was released into the culture medium as a core-like particle. We then examined how early core-like particles were released after recombinant virus infection. Sf9 cells were infected with recombinant baculovirus expressing $\mathrm{HCV}$ core protein and particle production was examined. As shown in Fig. 3, released core-like particles were detected as early as 2 days after infection, and efficiently released into the culture medium until 4 days after infection. We have not examined particle release more than 4 days after virus infection because wild type baculovirus-induced cytolysis occurs thereafter (Hwang et al., 1998).

Characterization of core-like particles To characterize the core-like particles, culture supernatants were harvested from recombinant baculovirus-infected cells and pelleted through the $30 \%$ sucrose cushion. The pellet was then further subjected to $\mathrm{CsCl}$ density gradient centrifugation. As shown in Fig. 4, core-like particles were detected in a specific fraction.

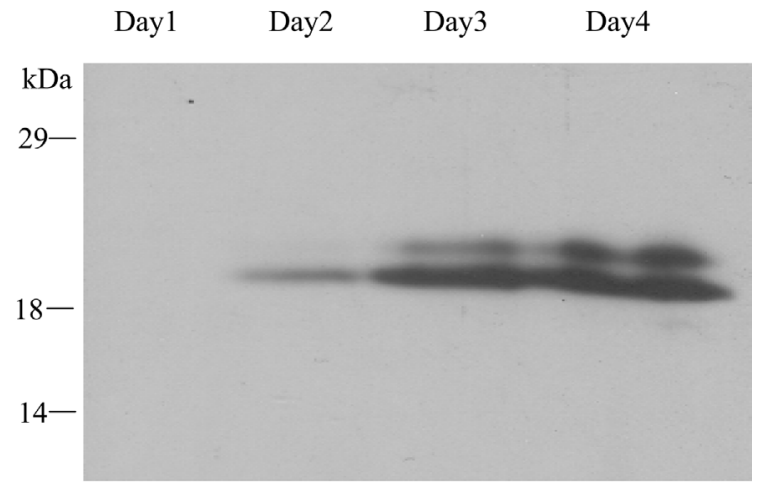

Fig. 3. Kinetics of core-like particle release from recombinant baculovirus-infected insect cells. Sf9 cells were infected with low m.o.i. of recombinant baculoviruses expressing core protein. Culture supernatants were harvested from day 1 to day 4 postinfection and partially purified core-like particles were detected by Western blot analysis.

We found that some particles were pelleted to the bottom in a $\mathrm{CsCl}$ gradient centrifugation (data not shown) but this pellet was no longer present after RNAse treatment $(0.1 \mathrm{mg} / \mathrm{ml})$, suggesting that some particles were associated with RNA. The density of $\mathrm{CsCl}$ fraction containing the core-like particles was approximately $1.25 \mathrm{~g} / \mathrm{ml}$. These particles were not observed in preparations from either core-E1-E2 recombinant virus- or wild type baculovirus-infected cells (data not shown). When the peak fractions were examined by electron microscopy, most of the released core proteins were heterogeneous in size with amorphous particle-like structure (data not shown). We 


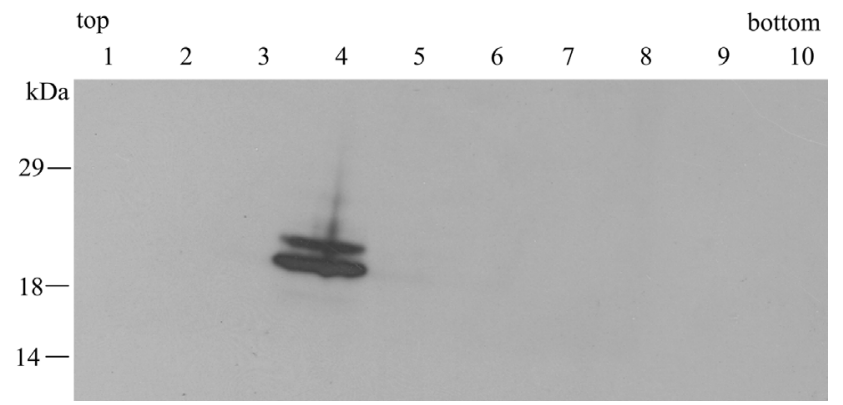

Fig. 4. Isolation of core-like particles by equilibrium density gradient. Sf9 cells were infected with recombinant baculoviruses expressing $\mathrm{HCV}$ core protein. The culture supernatant was collected at day 3 postinfection. Following removal of cell debris and recombinant baculoviruses, the particles were partially purified through a sucrose cushion and were subjected to equilibrium density gradient sedimentation. Ten fractions were collected and proteins were detected by Western blot analysis using HCV patient sera.

observed that some of the core proteins were easily aggregated during preparation for electron microscopy. However, we were unable to detect intracellular core-like particles.

Core-like particles contain HCV RNA To verify whether the core particles contain HCV RNA, RNA was extracted from supernatants purified from either the core- or the core-E1-E2expressing cells. To eliminate the possible DNA contamination, all RNA samples were treated with DNAse I for $30 \mathrm{~min}$ at $37^{\circ} \mathrm{C}$. RNA was then reverse transcribed and the cDNA was amplified by PCR. As shown in Fig. 5, cDNA corresponding to the HCV core RNA (573 nt) was detected (lane 2), indicating that core-like particles are associated with HCV RNA. However, the culture medium isolated from the core-E1-E2 construct did not contain HCV RNA (Fig. 5, lane 4).

\section{Discussion}

To understand the HCV morphogenesis, we studied the assembly of structural proteins in insect cells. Recombinant baculoviruses expressing $\mathrm{HCV}$ structural proteins were constructed and were used to infect Sf9 insect cells. Both virus-infected cells and culture supernatants were examined to see whether HCV structural proteins were capable of assembling into VLPs. VLPs were not detected in cells and supernatant. However, HCV core protein was released as core-like particles into the culture supernatant from recombinant virus expressing core protein alone. These corelike particles are associated with HCV RNA and amorphous in structure. We further found that these core-like particles were released into the medium as a membrane complex (data not shown). When core protein was expressed as a polyprotein with envelope protein(s), it was clear that particles were no

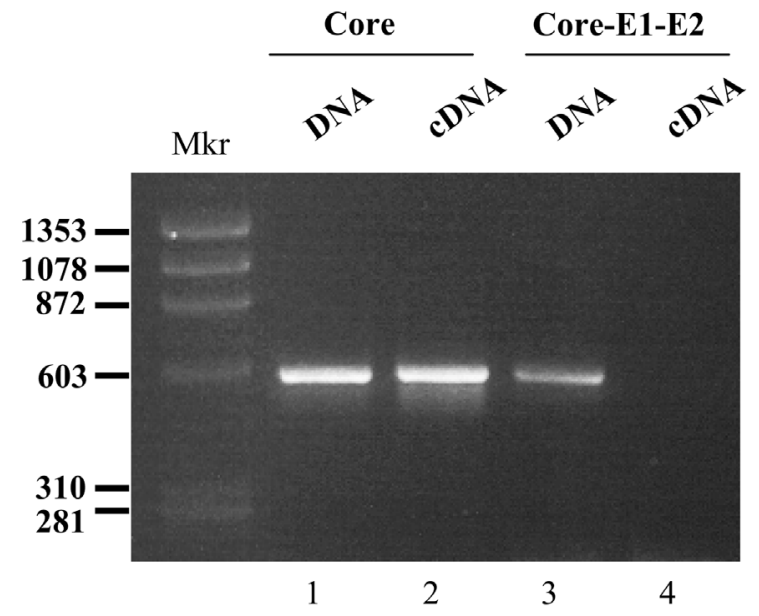

Fig. 5. Core-like particles contain HCV RNA. Sf9 insect cells were infected with recombinant baculoviruses expressing either HCV core or HCV core-E1-E2 protein. Culture supernatants were harvested at $60 \mathrm{~h}$ postinfection and were partially purified as described in Materials and Methods. RNA was extracted and reverse transcribed by AMV reverse transcriptase. The cDNA was amplified and then separated by an agarose gel. Mkr, molecular mass marker; lane 1, positive control for RT-PCR of core; lane 2, RT-PCR product from core-like particles; lane 3, positive control for RT-PCR of core-E1-E2 construct; lane 4, RTPCR product from culture supernatant from core-E1-E2.

longer released into the culture medium. Previously, it had been demonstrated that VLPs produced from recombinant baculoviruses expressing a part of the 5' UTR and structural proteins were retained in intracellular membrane vesicles and were not released into the culture medium (Baumert et al., 1998). At present, it is not clear why two systems show the discrepancy in particle formation. It has been reported that transmembrane domains of E1 and E2 function as retension signals in the endoplasmic reticulum (ER). Since core protein is colocalized with E2 protein (Santolini et al., 1994), it is possible that envelope proteins might have inhibitory effect on core particle release. Indeed, if envelope proteins were coexpressed, particle assembly was interrupted. The biological significance of $\mathrm{HCV}$ core release is not yet understood. HCV seems to employ a similar assembly mechanism to those of retroviruses and rhabdoviruses because assembly of gag protein of HIV-1 and simian immunodeficiency virus (Delchambre et al., 1989; Gheysen et al., 1989) and budding of rabies virus particle (Mebatsion et al., 1996) occurred in the absence of envelope proteins. Nevertheless, it is uncertain how virions are assembled in $\mathrm{HCV}$-infected patients.

How HCV core protein itself can be assembled into corelike particles in insect cells is not clear. Previously it has been reported that E1 and E2 hetrodimers do not leave the ER (Deleersnyder et al., 1997) and C-terminal 29 amino acids of E2 are responsible for the retention of E2 in the ER (Cocquerel et al., 1998). Based on this report, we made 
mutants consisting of either core-E1 (C-E1) or core-E1 but lacking $\mathrm{C}$-terminal hydrophobic region $\left(\mathrm{C}-\mathrm{E} 1_{340}\right)$. Therefore, these constructs do not contain E2 envelope protein. When these mutants were expressed in Sf9 cells, VLPs were still not released into the culture medium. In contrast, core protein alone was efficiently released out of the cells as early as 2 days after infection. These results suggest that HCV core has the intrinsic capacity to assemble into core-like particles. Since most of the cells were alive throughout the experimental period, the core-like particle release is not due to cytolysis. We also infected Sf9 cells with low titer of virus (m.o.i. of 3) to prevent cells from baculovirus-induced cytolysis (Hwang et al., 1998). Since HCV core protein was secreted from mammalian cell lines in culture (Sabile et al., 1999), and nonenveloped HCV nucleocapsids were overproduced in the plasma of HCV patients and released into the bloodstream (Maillard et al., 2001), our finding may represent a unique mechanism of nucleocapsid assembly of HCV. These data hence may represent a novel mechanism of HCV to evade the hosts immunity, thus contributing to the persistence of $\mathrm{HCV}$ infection.

Acknowledgments This study was supported by a grant from the Korean Ministry of Science and Technology (Korean Systems Biology Research Grant, M1-0309-06-0002) and by the research grant from Hallym University, Korea.

\section{References}

Baumert, T. F., Ito, S., Wong, D. T. and Liang, T. J. (1998) Hepatitis $\mathrm{C}$ virus structural proteins assemble into viruslike particles in insect cells. J. Virol. 72, 3827-3836.

Choo, Q.-L., Kuo, G., Weiner, A. J., Overby, L. R., Bradley, D. W. and Houghton, M. (1989) Isolation of a cDNA clone derived from a blood-borne non-A, non-B hepatitis genome. Science 244, 359-362.

Cocquerel, L., Meunier, J.-C., Pillez, A., Wychoeski, C. and Dubuisson, J. (1998) A retension signal necessary and sufficient for endoplasmic reticulum localization maps to the transmembrane domain of hepatitis $\mathrm{C}$ virus glycoprotein E2. $J$. Virol. 72, 2183-2191.

Crawford, S. E., Labbe, M., Cohen, J., Burroughs, M. H., Zhou, Y.-J. and Estes, M. K. (1994) Characterization of virus-like particles produced by the expression of rotavirus capsid protein in insect cells. J. Virol. 68, 5945-5952.

Delchambre, M., Gheysen, D., Thines, D., Thiriart, C., Jacobs, E., Verdin, E., Horth, M., Burny, A. and Bex, F. (1989) The GAG precursor of simian immunodeficiency virus assembles into virus-like particles. EMBO J. 8, 2653-2660.

Deleersnyder, V., Pillez, A., Wychowski, C., Blight, K., Xu, J., Hahn, Y. S., Rice, C. M. and Dubuisson, J. (1997) Formation of native hepatitis C virus glycoprotein complexes. J. Virol. 71, 697-704.

Gheysen, D., Jacobs, E., de Foresta, F., Thiriart, C., Francotte, M., Thines, D. and De Wilde, M. (1989) Assembly and release of HIV-1 precursor pr55gag virus-like particles from recombinant baculovirus-infected insect cells. Cell 59, 103-112.

Grakoui, A., Wychowski, C., Lin, C., Feinstone, S. M. and Rice, C. M. (1993) Expression and identification of hepatitis C virus polyprotein cleavage products. J. Virol. 67, 1385-1395.

Hijikata, M., Kato, N., Ootsuyama, Y., Nakagawa, M. and Shimotohno, K. (1991) Gene mapping of the putative structural region of the hepatitis $\mathrm{C}$ virus genome by in vitro processing analysis. Proc. Natl. Acad. Sci. USA 88, 5547-5551.

Hwang, S. B., Park, K.-J., Kim, Y.-S., Sung, Y. C. and Lai, M. M. C. (1997) Hepatitis C virus NS5B protein is a membraneassociated phosphoprotein with a predominantly perinuclear localization. Virology 227, 439-446.

Hwang, S. B. (1998) Expression and characterization of hepatitis $\mathrm{C}$ virus core proteins: effects of single amino acid substitution on protein conformation and subcellular localization. $J$. Biochem. Mol. Biol. 31, 281-286.

Hwang, S. B, Park, K.-J and Kim, Y. S. (1998) Overexpression of hepatitis delta antigen protects insect cells from baculovirusinduced cytolysis. Biochem. Biophys. Res. Commun. 244, 652658.

Inchauspe, G., Zebedee, S., Lee, D.-H., Sugitani, M., Nasoff, M. and Prince, A. M. (1991) Genomic structure of the human protype strain $\mathrm{H}$ of hepatitis $\mathrm{C}$ virus: Comparision with American and Japanese isolates. Proc. Natl. Acad. Sci. USA 88, 10292-10296.

Jiang, X., Wang, M., Graham, D. Y. and Estes, M. K. (1992) Expression, self-assembly, and antigenicity of the norwalk virus capsid protein. J. Virol. 66, 6527-6532.

Kato, N., Hijikata, M., Ootsuyama, Y., Nakagawa, M., Ohkoshi, S., Sugimura, T. and Shimotohno, K. (1990) Molecular cloning of the human hepatitis $\mathrm{C}$ virus genome from Japanese patients with non-A, non-B hepatitis. Proc. Natl. Acad. Sci. USA 87, 9524-9528.

Kim, J. S., Ryu, J., Hwang, S. B., Lee, S. Y., Choi, S. Y. and Park, J. (2004) Suppression of ceramide-induced cell death by hepatitis C virus core protein. J. Biochem. Mol. Biol. 37, 192198.

Lin, C., Lindenbach, B. D., Pragai, B., McCourt, D. W. and Rice, C. M. (1994) Processing of the hepatitis C E2-NS2 region: identification of $\mathrm{p} 7$ and two distinct E2-specific products with different C termini. J. Virol. 68, 5063-5073.

Luo, L., Li, Y. and Kang, C. Y. (1990) Expression of gag precursor protein and secretion of virus-like gag particles of HIV-2 from recombinant baculovirus-infected insect cells. Virology 179, 874-880.

Maillard, P., Krawczynski, K., Nitkiewicz, J., Bronnert, C., Sidorkiewicz, M., Gounon, P., Dubuisson, J., Faure, G., Crainic, R. and Budkowska, A. (2001) Nonenveloped nucleocapsids of hepatitis $\mathrm{C}$ virus in the serum of infected patients. J. Virol. 75, 8240-8250.

Mebatsion, T., Konig, M. and Conzelmann, K.-K. (1996) Budding of rabies virus particles in the absence of the spike glycoprotein. Cell 84, 941-951.

Miller, R. H. and Purcell, R. H. (1990) Hepatitis C virus shares amino acids sequence similarity with pestiviruses and flaviviruses as well as members of two plant virus super groups. Proc. Natl. Acad. Sci. USA 87, 2057-2061.

Park, K. J., Choi, S. H., Koh, M. S., Kim, S. W. and Hwang, S. B. (2000) Identification of a cellular protein interacting with RNA polymerase of hepatitis C virus. J. Biochem. Mol. Biol. 
33, 59-62.

Sabile, A., Perlemuter, G., Bono, F., Kohara, K., Demaugre, F., Kohara, M., Matsuura, Y., Miyamura, T., Brechot, C. and Barba, G. (1999) Hepatitis C virus core protein binds to apolipoprotein AII and its secretion is modulated by fibrates. Hepatol. 30, 1064-1076.

Saito, I., Miyamura, T., Ohbayashi, A., Harada, H., Katayama, T., Kikuchi, S., Watanabe, Y., Koi, S., Orji, M., Ohta, Y., Choo, Q.-L., Houghton, M. and Kuo, G. (1990) Hepatitis C virus infection is associated with the development of hepatocellular carcinoma. Proc. Natl. Acad. Sci. USA 87, 6547-6549.

Santolini, E., Migliaccio, G. and La Monica, N. (1994)
Biosynthesis and biochemical properties of the hepatitis $\mathrm{C}$ virus core protein. J. Virol. 68, 3631-3641.

Shimotohno, K. (1993) Hepatocellular carcinoma in Japan and its linkage to infection with hepatitis C virus. Semin. Virol. 4, 305312.

Takamizawa, A., Mori, C., Fuke, I., Manabe, S., Murakami, S., Fujita, J., Onishi, E., Andoh, T., Yoshida, I. and Okayama, H. (1991) Structure and organization of the hepatitis C virus genome isolated from human carriers. J. Virol. 65, 1105-1113.

Wang, C. and Siddiqui, A. (1995) Structure and function of the hepatitis C virus internal ribosome entry site. Curr. Topics Microbiol. Immunol. 203, 99-115. 